\title{
A New Approach to the Existence of Quasiperiodic Solutions for Second-Order Asymmetric $p$-Laplacian Differential Equations
}

\author{
Xiaoming Wang $\mathbb{D}^{1}$ and Lixia Wang ${ }^{2}$ \\ ${ }^{1}$ School of Mathematics \& Computer Science, Shangrao Normal University, Shangrao 334001, China \\ ${ }^{2}$ School of Sciences, Tianjin Chengjian University, Tianjin 300384, China \\ Correspondence should be addressed to Xiaoming Wang; wxmsuda03@163.com
}

Received 24 April 2018; Accepted 17 May 2018; Published 19 June 2018

Academic Editor: Liguang Wang

Copyright (c) 2018 Xiaoming Wang and Lixia Wang. This is an open access article distributed under the Creative Commons Attribution License, which permits unrestricted use, distribution, and reproduction in any medium, provided the original work is properly cited.

For $p \geq 2$ and $\phi_{p}(s):=|s|^{p-2} s$, we propose a new estimate approach to study the existence of Aubry-Mather sets and quasiperiodic solutions for the second-order asymmetric $p$-Laplacian differential equations $\left(\phi_{p}\left(x^{\prime}\right)\right)^{\prime}+\lambda \phi_{p}\left(x^{+}\right)-\mu \phi_{p}\left(x^{-}\right)=\psi(t, x)$, where $\lambda$ and $\mu$ are two positive constants satisfying $\lambda^{-1 / p}+\mu^{-1 / p}=2 / \omega$ with $\omega \in \mathbb{R}^{+}, \psi(t, x) \in C^{0,1}\left(\mathbf{S}^{p} \times \mathbb{R}\right)$ is a continuous function, $2 \pi_{p^{-}}$ periodic in the first argument and continuously differentiable in the second one, $x^{ \pm}=\max \{ \pm x, 0\}, \pi_{p}=2 \pi(p-1)^{1 / p} / p \sin (\pi / p)$, and $S^{p}=\mathbb{R} / 2 \pi_{p} \mathbb{Z}$. Using the Aubry-Mather theorem given by Pei, we obtain the existence of Aubry-Mather sets and quasiperiodic solutions under some reasonable conditions. Particularly, the advantage of our approach is that it not only gives a simpler estimation procedure, but also weakens the smoothness assumption on the function $\psi(t, x)$ in the existing literature.

\section{Introduction}

In recent years, all kinds of nonlinear dynamic behavior, such as the existence of positive solutions [1-16] and signchanging solutions $[17,18]$, the existence and uniqueness of solutions [19-25], the existence and multiplicity results [26-30], and the existence of unbounded solutions[31,32], have been widely investigated for some nonlinear ordinary differential equations and partial differential equations due to the application in many fields such as physics, mechanics, and the engineering technique fields. In the present paper, we deal with the existence of Aubry-Mather sets and quasiperiodic solutions for the second-order differential equations with a $p$-Laplacian and an asymmetric nonlinear term

$$
\left(\phi_{p}\left(x^{\prime}\right)\right)^{\prime}+\lambda \phi_{p}\left(x^{+}\right)-\mu \phi_{p}\left(x^{-}\right)=\psi(t, x)
$$

where $\phi_{p}(s):=|s|^{p-2} s, p \geq 2, x^{ \pm}=\max \{ \pm x, 0\}$, and $\lambda$ and $\mu$ are positive constants satisfying

$$
\frac{1}{\lambda^{1 / p}}+\frac{1}{\mu^{1 / p}}=\frac{2}{\omega}, \quad \omega \in \mathbb{R}^{+}
$$

where $\psi(t, x) \in C^{0,1}\left(\mathbf{S}^{p} \times \mathbb{R}\right)$ is a continuous function, $2 \pi_{p^{-}}$ periodic in the first argument and continuously differentiable in the second one, where $S^{p}=\mathbb{R} / 2 \pi_{p} \mathbb{Z}$. Since the pioneering works of Aubry [33] and Mather [34], the existence of AubryMather sets and quasiperiodic solutions for a variety of differential equations, such as Hamiltonian systems [35-41], and reversible systems [42-44] had been widely investigated due to the application in many fields such as one-dimensional crystal model of solid state physics, differential geometry, and dynamical systems (see $[45,46]$ ).

If $p=2$, then $\pi_{p}=\pi$ and (1) reduces to the following piecewise linear equation:

$$
x^{\prime \prime}+\lambda x^{+}-\mu x^{-}=\psi(t, x)
$$

and (2) becomes

$$
\frac{1}{\sqrt{\lambda}}+\frac{1}{\sqrt{\mu}}=\frac{2}{\omega}, \quad \omega \in \mathbb{R}^{+} .
$$

The first result is due to Capietto and Liu [38], who proved that the existence of Aubry-Mather sets and quasiperiodic solutions of (3) for some $\omega \in \mathbb{Q}^{+}$in (4), provided that 
$\psi(t, x)=e(t)-\phi(x), e(t) \in C^{2}(\mathbb{R} / 2 \pi \mathbb{Z})$, and the perturbation term $\phi(x) \in C^{2}(\mathbb{R}) \cap L^{\infty}(\mathbb{R})$ satisfies some growth conditions. Recently, this result was extended to a much weaker smoothness nonlinearity $\psi(t, x)$. In [41], by using the Aubry-Mather theorem generalized by Pei [37], the present author [41] studied the existence of Aubry-Mather sets and quasiperiodic solutions of (3), under the condition that $\omega \in$ $\mathbb{R}^{+}$in $(4)$ and $\psi(t, x) \in C^{0,1}\left(\mathbf{S}^{p} \times \mathbb{R}\right)$ can be allowed to be either a bounded function or an unbounded function, which differs from above existing results.

In [39], (3) has been generalized to the following $p$ Laplacian-like nonlinear differential equation:

$$
\begin{aligned}
& (p-1)^{-1}\left(\phi_{p}\left(x^{\prime}\right)\right)^{\prime}+\lambda \phi_{p}\left(x^{+}\right)-\mu \phi_{p}\left(x^{-}\right)+g(x) \\
& \quad=h(t)
\end{aligned}
$$

where $p>1, \lambda$ and $\mu$ are positive constants satisfying (2) with $\omega=n \in \mathbb{N}, g(x) \in C^{1}(\mathbb{R}) \cap L^{\infty}(\mathbb{R})$, and $h(t) \in C^{2}\left(\mathbf{S}^{p}\right)$ is a $2 \pi_{p^{-}}$ periodic function. They considered the existence of AubryMather sets and quasiperiodic solutions of (5) when $g(x)$ satisfies some further approximate properties at infinity. We notice that in [39], to overcome the barriers of weak smoothness, they made use of exchange of the role of time and angle variables skills and showed the existence of Aubry-Mather sets and quasiperiodic solutions by employing a version of Aubry-Mather theorem obtained by Pei [37]. Moreover, the results in [39] need the smoothness requirement of the perturbation function at least to $C^{2}$ smooth in $t$.

Now a natural question to ask is whether the smoothness of the function $h(t)$ in (5) is further reduced; we can also obtain the same results as [13]. In this paper, we will deal with this interesting problem and answer this question in the form of Theorem 1 with more general case (1) than that of (5). Because of the presence of weak smoothness nonlinearity, the methods of seeking the existence of Aubry-Mather sets and quasiperiodic solutions for problems as [38, 39] do not seem to be applicable to (1). This phenomenon provokes some mathematical difficulties, which make the study of (1) particularly interesting. Our approach here is mainly based on the direct proof of the Poincaré map of the transformed system satisfying monotone twist property and is developed from the present author (see the recent papers [41, 44]) but is more subtle than the ones in [38-40]. More efforts have to be made to estimate the monotone twist property for the Poincaré map of the transformed system, but the procedure is a little simpler than those in [38-40]. One important advantage of our approach is that it does not require any high smoothness assumptions on function $\psi(t, x)$. Our results improve and generalize some results of the previous studies $[39,41]$ to some extent.

The main result of this paper is the following theorem.

Theorem 1. Suppose that (2) holds. Moreover, $\psi(t, x) \in$ $C^{0,1}\left(\mathbf{S}^{p} \times \mathbb{R}\right)$ satisfies the following conditions:

$\left(A_{1}\right)$ The limit is

$$
\lim _{|x| \rightarrow+\infty} \psi_{x}(t, x)=0, \quad \text { uniformly in } t \in\left[0,2 \pi_{p}\right] .
$$

\section{$\left(A_{2}\right)$ There exist constants $d \geq 0, \beta>0$, such that}

$$
\begin{aligned}
& \operatorname{sgn}(x)\left[(p-1) \psi(t, x)-x \psi_{x}(t, x)\right]>\beta, \\
& \qquad \text { for } \forall|x| \geq d \text { and } \forall t \in\left[0,2 \pi_{p}\right] .
\end{aligned}
$$

Then there exists $\varepsilon_{0}>0$, such that, for any $\alpha \in\left(2 \omega \pi_{p}\right.$, $\left.2 \omega \pi_{p}+\varepsilon_{0}\right)$, (1) possesses an Aubry-Mather type solution $z_{\alpha}(t)=$ $\left(x_{\alpha}(t), x_{\alpha}^{\prime}(t)\right)$ with rotation number $\alpha$; that is,

(i) if $\alpha=k / m$ is rational and $(k, m)=1$, the solutions $z_{\alpha}^{i}(t)=z_{\alpha}\left(t+2 \pi_{p} i\right), 0 \leq i \leq m-1$, are mutually unlinked periodic solutions of period $m$;

(ii) if $\alpha$ is irrational, the solution $z_{\alpha}(t)$ is either a usual quasiperiodic solution or a generalized one.

Remark 2. A solution is called generalized quasiperiodic one if the closed set

$$
M_{\alpha} \equiv \overline{\left\{z_{\alpha}\left(2 \pi_{p} i\right), i \in \mathbf{Z}\right\}}
$$

is Denjoy's minimal set (see its definition in [47]).

Remark 3. Using the rule of L'Hospital to condition $\left(A_{1}\right)$, it can easily be seen that

$$
\lim _{|x| \rightarrow+\infty} \frac{\psi(t, x)}{x}=0, \quad \text { uniformly in } t \in\left[0,2 \pi_{p}\right] .
$$

Remark 4. We noticed that the perturbations $g(x)$ and $h(t)$ in [39] need to be bounded. But from $\left(A_{1}\right)$ and $\left(A_{2}\right)$ of this paper, it is easy to verify that the perturbation $\psi(t, x)$ can be either a bounded function or an unbounded function. For example, we can set $\psi(t, x)$ to be a bounded function $\arctan x \cdot\left(1+\sin ^{2}\left(\left(\pi / \pi_{p}\right) t\right)\right)$ or an unbounded function $x^{1 / 3} \cdot\left(1+\cos ^{2}\left(\left(\pi / \pi_{p}\right) t\right)\right)$ for $t \in\left[0,2 \pi_{p}\right]$ when $d=1$ and $\beta=\pi / 4-1 / 2$ in Theorem 1 . Moreover, positive constant $\omega=n \in \mathbb{N}$ satisfying (1.2) in [39] has been extended to the case $\omega \in \mathbb{R}^{+}$in this paper. Thus, our situation is more general than the results obtained in [39] for $p \geq 2$.

Remark 5. If $p=2$, let us point out that the results in Theorem 1 have covered the conclusions obtained by Wang [41]. Besides, the estimation process in this paper is much more meticulous than that in [41] since the $p$-Laplacian $\left(\phi_{p}\left(x^{\prime}\right)\right)^{\prime}$ of a function $x(t)$, with $p>2$, is no longer linear. Therefore, the results obtained in this paper are natural generalizations and refinements of the results obtained in [41].

The main idea of our proof is acquired from [39, 41]. The proof of Theorem 1 is based on an Aubry-Mather theorem due to Pei [37]. The rest of this manuscript is as follows. In Section 2, we introduce some action-angle variables transformation to transform system (1) into an equivalent integral Hamiltonian system and then present some growth properties on the corresponding action and angle variables functions. In Section 3, we provide some crucial estimates by some lemmas which say that the Poincaré mapping of the new system is monotone twist around the infinity. At last, Section 4 gives the proof of Theorem 1 by using Pei's AubryMather theorem [37]. 


\section{Preliminaries}

2.1. The Action and Angle Variables. Let $S_{p}(t)=\sin _{p} t$ be the solution of

$$
\left(\phi_{p}\left(u^{\prime}\right)\right)^{\prime}+\phi_{p}(u)=0
$$

satisfying the initial condition $u(0)=0, u^{\prime}(0)=1$. Then it follows from [48] that $S_{p}(t)=\sin _{p} t$ is a $2 \pi_{p}$-periodic $C^{2}$ odd function with $\sin _{p}\left(\pi_{p}-t\right)=\sin _{p} t$, for $t \in\left[0, \pi_{p} / 2\right]$, and $\sin _{p}\left(2 \pi_{p}-t\right)=-\sin _{p} t$ for $t \in\left[\pi_{p}, 2 \pi_{p}\right]$. Moreover, for $t \in$ $\left(0, \pi_{p} / 2\right), S_{p}(t)>0, S_{p}^{\prime}(t)>0$ and $S_{p}:\left[0, \pi_{p} / 2\right] \rightarrow[0,(p-$ $1)^{1 / p}$ ] can be implicitly given by

$$
\int_{0}^{\sin _{p} t} \frac{d s}{\left(1-s^{p} /(p-1)\right)^{1 / p}}=t .
$$

Introducing a new variable $y=-\phi_{p}\left(x^{\prime}\right)$, then (9) is equivalent to the planar system

$$
\begin{aligned}
& x^{\prime}=-\phi_{q}(y), \\
& y^{\prime}=\phi_{p}(x),
\end{aligned}
$$

where $q$ is the conjugate exponent of $p: p^{-1}+q^{-1}=1$. Letting $(x, y)=\left(C_{p}(t), S_{p}(t)\right)$ be the unique solution of (11) satisfying $\left(C_{p}(0), S_{p}(0)\right)=(1,0)$, then the functions $C_{p}(t)$ and $S_{p}(t)$ are much similar to cosine and sine. It follows from [49] that $C_{p}(t) \in C^{2}$ and $S_{p}(t) \in C^{1}$ are $2 \pi_{p}$-periodic, and for $\forall n \in \mathbb{Z}$, $C_{p}(t)=0$ iff $t=\pi_{p} / 2+n \pi_{p}$, and $S_{p}(t)=0$ iff $t=n \pi_{p}$. Moreover, $C_{p}^{\prime}(t)=-\phi_{q}\left(S_{p}(t)\right)$ and $S_{p}^{\prime}(t)=\phi_{p}\left(C_{p}(t)\right)$, and $(1 / p)\left|C_{p}(t)\right|^{1 / p}+(1 / q)\left|S_{p}(t)\right|^{1 / q} \equiv 1 / p$.

Now we consider (1). Set $y=-\phi_{p}\left(x^{\prime}\right)$ in (1); then (1) can be rewritten as a planar system

$$
\begin{aligned}
& x^{\prime}=-\phi_{q}(y), \\
& y^{\prime}=\lambda \phi_{p}\left(x^{+}\right)-\mu \phi_{p}\left(x^{-}\right)-\psi(t, x),
\end{aligned}
$$

where $q=p /(p-1)$ is the conjugate exponent of $p$.

Lemma 6. For $p \geq 2$ and for any $\left(x_{0}, y_{0}\right) \in \mathbb{R}^{2}, t_{0} \in \mathbb{R}$, the solution

$$
z(t)=\left(x\left(t, t_{0}, x_{0}, y_{0}\right), y\left(t, t_{0}, x_{0}, y_{0}\right)\right)
$$

of (12) satisfying the initial condition $z\left(t_{0}\right)=\left(x_{0}, y_{0}\right)$ is unique and exists on the whole $t$-axis.

Proof. The proof of uniqueness can be established similarly to the proof of Proposition 2 in [50]; the global existence result can be acquired similarly to Lemma 3.1 in [51].

Let $(C(t), S(t))$ be the solution of the following homogeneous system:

$$
\begin{aligned}
x^{\prime} & =-\phi_{q}(y), \\
y^{\prime} & =\lambda \phi_{p}\left(x^{+}\right)-\mu \phi_{p}\left(x^{-}\right), \\
x(0) & =1, \\
y(0) & =0 .
\end{aligned}
$$

Then, by using (2) and direct computation, one obtains the following.

Lemma 7. (i) Both $C(t) \in C^{2}$ and $S(t) \in C^{1}$ are $2 \pi_{p} / \omega$ periodic functions, and $C(t)$ can be given by

$C(t)$

$$
= \begin{cases}C_{p}\left(\lambda^{1 / p} t\right), & 0 \leq|t| \leq \frac{\pi_{p}}{2 \lambda^{1 / p}}, \\ C_{p}\left(\mu^{1 / p}\left(t-\frac{\pi_{p}}{2 \lambda^{1 / p}}+\frac{\pi_{p}}{2 \mu^{1 / p}}\right)\right), & \frac{\pi_{p}}{2 \lambda^{1 / p}} \leq t \leq \frac{\pi_{p}}{\omega} .\end{cases}
$$

(ii) $C^{\prime}(t)=-\phi_{q}(S(t))$ and $S^{\prime}(t)=\lambda \phi_{p}\left(C(t)^{+}\right)-$ $\mu \phi_{p}\left(C(t)^{-}\right)$.

(iii) $(1 / q)|S(t)|^{q}+(1 / p)\left(\lambda\left|C(t)^{+}\right|^{p}+\mu\left|C(t)^{-}\right|^{p}\right)=\lambda / p$.

Now we introduce an action-angle variables transformation by the mapping $\Psi: \mathbb{S}^{p} \times(0,+\infty) \rightarrow \mathbb{R}^{2} \backslash\{(0,0)\}$, where $(x, y)=\Psi(\theta, I)$ defined by the formula

$$
\begin{aligned}
& x=(\gamma I)^{1 / p} C\left(\frac{\theta}{\omega}\right), \\
& y=(\gamma I)^{1 / q} S\left(\frac{\theta}{\omega}\right),
\end{aligned}
$$

where $\gamma=\lambda^{-1} \omega p$ is a constant. This transformation is said to be a generalized symplectic transformation because its Jacobian is equal to 1 .

2.2. Some Properties on Action and Angle Variables Functions. Under the transformation $\Psi$ and using Lemma 7 (iii), (12) is changed into

$$
\begin{aligned}
& \dot{\theta}=\Phi_{1}(t, \theta, I), \\
& \dot{I}=\Phi_{2}(t, \theta, I),
\end{aligned}
$$

where $\Phi_{1}(t, \theta, I)=\omega+x(\theta, I) \psi(t, x(\theta, I)) / p I, \Phi_{2}(t, \theta, I)=$ $\phi_{q}(y(\theta, I)) \psi(t, x(\theta, I)) / \omega$.

We notice that the relation between (17) and (12) is that if $\theta(t)=\theta\left(t ; \theta_{0}, I_{0}\right), I(t)=I\left(t ; \theta_{0}, I_{0}\right)$ are the solutions of $(17)$ with the initial value condition $\theta(0)=\theta_{0}, I(0)=I_{0}$, then

$$
\begin{aligned}
x(t) & =x\left(t ; \theta_{0}, I_{0}\right)=x\left(\theta\left(t ; \theta_{0}, I_{0}\right), I\left(t ; \theta_{0}, I_{0}\right)\right) \\
& =\left(\gamma I\left(t ; \theta_{0}, I_{0}\right)\right)^{1 / p} C\left(\frac{\theta\left(t ; \theta_{0}, I_{0}\right)}{\omega}\right)
\end{aligned}
$$

and

$$
\begin{aligned}
y(t) & =y\left(t ; \theta_{0}, I_{0}\right)=y\left(\theta\left(t ; \theta_{0}, I_{0}\right), I\left(t ; \theta_{0}, I_{0}\right)\right) \\
& =\left(\gamma I\left(t ; \theta_{0}, I_{0}\right)\right)^{1 / q} S\left(\frac{\theta\left(t ; \theta_{0}, I_{0}\right)}{\omega}\right)
\end{aligned}
$$

are the solutions of (12) with initial data $x(0)=$ $x\left(0 ; \theta_{0}, I_{0}\right), y(0)=y\left(0 ; \theta_{0}, I_{0}\right)$. By Lemma 6 , (17) has a unique solution for $I_{0}>0$ and $\theta_{0} \in \mathbb{R}$. Moreover, this 
solution has continuous derivatives with respect to initial data $\theta_{0}$ and $I_{0}$.

For notional convenience, hereinafter, we write $x, y, \theta, I$ instead of $x\left(\theta\left(t ; \theta_{0}, I_{0}\right), I\left(t ; \theta_{0}, I_{0}\right)\right), y\left(\theta\left(t ; \theta_{0}, I_{0}\right), I\left(t ; \theta_{0}, I_{0}\right)\right)$, $\theta\left(t ; \theta_{0}, I_{0}\right), I\left(t ; \theta_{0}, I_{0}\right)$, respectively. ing.

Firstly, by some simple calculations, we have the follow-

Lemma 8. (i) $\partial x / \partial I=x / p I, \quad \partial y / \partial I=y / q I, \partial x / \partial \theta=$ $-(1 / \omega) \phi_{q}(y), \partial y / \partial \theta=\left(\lambda \phi_{p}\left(x^{+}\right)-\mu \phi_{p}\left(x^{-}\right)\right) / \omega$.

(ii) $|C(t)| \leq \max \left\{1,(\lambda / \mu)^{1 / p}\right\}:=C_{\infty},|S(t)| \leq(q \lambda /$ $p)^{1 / q}:=S_{\infty}$.

Now we are concerned with the growth estimates with regard to $I\left(t ; \theta_{0}, I_{0}\right)$ and $\theta\left(t ; \theta_{0}, I_{0}\right)$.

Lemma 9. The limit

$$
\lim _{I_{0} \rightarrow+\infty} I\left(t ; \theta_{0}, I_{0}\right)=+\infty
$$

holds uniformly on $t \in\left[0,2 \pi_{p}\right]$.

Proof. In view of $\left(\psi_{0}\right)$ and (16), there exist constants $D>0$, $K>0$, such that

$$
\begin{array}{r}
\left|I^{\prime}(t)\right|=\left|\frac{\phi_{q}(y(\theta, I)) \psi(t, x(\theta, I))}{\omega}\right| \leq D I(t)+K, \\
\forall I \neq 0 .
\end{array}
$$

Then, by the Gronwall inequality, one has

$$
\begin{gathered}
e^{-2 \pi D} I_{0}-\frac{K}{D}\left(1-e^{-2 \pi D}\right) \leq I(t) \\
\leq e^{2 \pi D} I_{0}+\frac{K}{D}\left(e^{2 \pi D}-1\right)
\end{gathered}
$$

for all $t \in\left[0,2 \pi_{p}\right]$.

So, by (22), $I\left(t ; \theta_{0}, I_{0}\right) \rightarrow+\infty$ as $I_{0} \rightarrow+\infty$ uniformly for $t \in\left[0,2 \pi_{p}\right]$.

According to (22), it is easy to see the following.

Corollary 10. $\forall \theta_{0} \in \mathbb{R}$ and $\forall t \in\left[0,2 \pi_{p}\right]$, there exist constants $\rho_{2}>\rho_{1}>0$ and $\bar{I}>0$, such that

$$
\rho_{1} I_{0} \leq I\left(t ; \theta_{0}, I_{0}\right) \leq \rho_{2} I_{0}
$$

when $I_{0} \geq \bar{I}$.

Lemma 11. $\forall \theta_{0} \in \mathbb{R}$ and $\forall t \in\left[0,2 \pi_{p}\right]$, there exists constant $\bar{I}>0$, such that

$$
\frac{\omega}{2} \leq \theta^{\prime}\left(t ; \theta_{0}, I_{0}\right) \leq 2 \omega
$$

if $I_{0} \geq \bar{I}$.

Proof. Since $\left(\psi_{0}\right)$ holds, then, for every $\varepsilon>0$, there exists $M=M(\varepsilon)>0$, such that

$$
|\psi(t, x)| \leq \varepsilon|x|
$$

if $|x| \geq M$ and $\forall t \in\left[0,2 \pi_{p}\right]$. Hence,

$$
\frac{d \theta}{d t}=\omega+\frac{x \psi(t, x)}{p I} \geq \omega-\frac{\varepsilon x^{2}}{p I} .
$$

Thus, by using action-angle variables transformation (16) and Lemma 9, there exists $\overline{I_{1}}>0$ such that $d \theta / d t \geq \omega / 2$ if $I_{0} \geq \overline{I_{1}}$.

For if $|x| \leq M$, we assume that $|\psi(t, x)| \leq \psi_{\infty}$, where $\psi_{\infty}=\max \left\{|\psi(t, x)|: t \in\left[0,2 \pi_{p}\right],|x| \leq M\right\}$, and then

$$
\frac{d \theta}{d t}=\omega+\frac{x \psi(t, x)}{p I} \geq \omega-\frac{\psi_{\infty}|x|}{p I} .
$$

So, by (16), Lemma 8 (ii), and Lemma 9, there exists a constant $\overline{I_{2}}>0$, such that $d \theta / d t \geq \omega / 2$ if $I_{0} \geq \overline{I_{2}}$.

If we choose $\bar{I}=\max \left\{\overline{I_{1}}, \overline{I_{2}}\right\}$, then $I_{0} \geq \bar{I}$ implies $d \theta / d t \geq$ $\omega / 2$.

Exploiting the same arguments, one can show that the inequality on the right side of (i) holds.

\section{Twist Property and Proof of Theorem 1}

Let the Poincaré mapping $P$ of equation (17) be

$$
P:\left(\theta_{0}, I_{0}\right) \longmapsto\left(\theta\left(2 \pi_{p}, \theta_{0}, I_{0}\right), I\left(2 \pi_{p}, \theta_{0}, I_{0}\right)\right) .
$$

In order to apply the Aubry-Mather theorem developed by Pei [37], we only need to show that the Poincaré mapping $P$ is a monotone twist map around the infinity; that is, it is enough to show $\partial \theta\left(2 \pi ; \theta_{0}, I_{0}\right) / \partial I_{0}<0$ if $I_{0} \gg 1$. In the following we are going to give its detailed proofs by some lemmas.

Similarly, for notional convenience, hereinafter, we also write $x, y, \theta, I$ instead of $x\left(\theta\left(t ; \theta_{0}, I_{0}\right), I\left(t ; \theta_{0}, I_{0}\right)\right)$, $y\left(\theta\left(t ; \theta_{0}, I_{0}\right), I\left(t ; \theta_{0}, I_{0}\right)\right), \theta\left(t ; \theta_{0}, I_{0}\right), I\left(t ; \theta_{0}, I_{0}\right)$, respectively.

Lemma 12. The following convergences hold uniformly on $t \in$ $\left[0,2 \pi_{p}\right]$ :

(i) $x \psi(t, x) / I \rightarrow 0 ; x^{2} \psi_{x}(t, x) / I \rightarrow 0$, as $I_{0} \rightarrow+\infty$.

(ii) $\phi_{q}(y) \psi(t, x) / I \rightarrow 0 ; \phi_{q}(y) x \psi_{x}(t, x) / I \rightarrow 0$, as $I_{0} \rightarrow$ $+\infty$.

(iii) $x \psi^{2}(t, x) \phi_{q}^{\prime}(y) \phi_{p}(x) / I^{2} \rightarrow 0 ; x \psi(t, x) \psi_{x}(t, x) \phi_{q}^{2}(y) /$ $I^{2} \rightarrow 0 ; x^{2} \psi_{x}(t, x) \psi(t, x) \phi_{q}^{\prime}(y) \phi_{p}(x) / I^{2} \rightarrow 0 ;$ $x^{2} \psi_{x}^{2}(t, x) \phi_{q}^{2}(y) / I^{2} \rightarrow 0$, as $I_{0} \rightarrow+\infty$.

Proof. If $\left(A_{1}\right)$ and $\left(\psi_{0}\right)$ hold, then to each $\varepsilon>0$ there corresponds a positive number $M=M(\varepsilon)>0$, such that

$$
\left|\psi_{x}(t, x)\right| \leq \frac{\varepsilon}{2 \gamma^{2 / p}}
$$

and

$$
|\psi(t, x)| \leq \frac{\varepsilon}{2 \gamma^{2 / p}}|x|
$$

when $|x| \geq M$ and $t \in\left[0,2 \pi_{p}\right]$, where $\gamma=\lambda^{-1} \omega p$ is a constant given in (16). 
Denote $V_{1}(\varepsilon)=\max \left\{|\psi(t, x)|: t \in\left[0,2 \pi_{p}\right],|x| \leq M\right\}$, $V_{2}(\varepsilon)=\max \left\{\left|\psi_{x}(t, x)\right|: t \in\left[0,2 \pi_{p}\right],|x| \leq M\right\}$.

(i) By action-angle variables transformation (16) and Lemma 8 (ii) and $p \geq 2$, we have

$$
\begin{aligned}
\left|\frac{x \psi(t, x)}{I}\right| & \leq \frac{M V_{1}(\varepsilon)}{I}+\frac{\varepsilon x^{2}}{2 \gamma^{2 / p I}} \\
& \leq \frac{M V_{1}(\varepsilon)}{I}+\frac{\varepsilon}{2 I^{1-2 / p}} \\
\left|\frac{x^{2} \psi_{x}(t, x)}{I}\right| & \leq \frac{M^{2} V_{2}(\varepsilon)}{I}+\frac{\varepsilon x^{2}}{2 \gamma^{2 / p} I} \\
& \leq \frac{M^{2} V_{2}(\varepsilon)}{I}+\frac{\varepsilon}{2 I^{1-2 / p}} .
\end{aligned}
$$

Then, given $\bar{I}>0$, choosing $I_{0}$ so that $I_{0} \geq \bar{I}$, by using Corollary 10, provided

$$
I(t) \geq \max \left\{\frac{2 M V_{1}(\varepsilon)}{\varepsilon} ; \frac{2 M^{2} V_{2}(\varepsilon)}{\varepsilon} ; 1\right\},
$$

we have

$$
\begin{gathered}
\left|\frac{x \psi(t, x)}{I}\right| \leq \frac{\varepsilon}{2}+\frac{\varepsilon}{2} \leq \varepsilon \\
\left|\frac{x^{2} \psi_{x}(t, x)}{I}\right| \leq \frac{\varepsilon}{2}+\frac{\varepsilon}{2} \leq \varepsilon
\end{gathered}
$$

Since $\varepsilon>0$ is arbitrary, the proof of (i) is complete.

For (ii), observe that $p \geq 2$, and, combining (16) and Lemma 8 (ii), one has

$$
\begin{aligned}
\left|\frac{\phi_{q}(y) \psi(t, x)}{I}\right| \leq & \frac{\varepsilon|y|^{q-1}|x|}{2 \gamma^{2 / p} I}+\frac{V_{1}(\varepsilon)|y|^{q-1}}{I} \\
\leq & \frac{\varepsilon}{2} C_{\infty} S_{\infty}^{q-1} I^{2 / p-1} \\
& +V_{1}(\varepsilon) \gamma^{1 / p} S_{\infty}^{q-1} I^{-1 / q} \\
\left|\frac{\phi_{q}(y) x \psi_{x}(t, x)}{I}\right| \leq & \frac{\varepsilon|y|^{q-1}|x|}{2 \gamma^{2 / p} I}+\frac{M|y|^{q-1} V_{2}(\varepsilon)}{I} \\
\leq & \frac{\varepsilon}{2} C_{\infty} S_{\infty}^{q-1} I^{2 / p-1} \\
& +M \gamma^{1 / p} V_{2}(\varepsilon) S_{\infty}^{q-1} I^{-1 / q} .
\end{aligned}
$$

Then, given $\bar{I}>0$, choosing $I_{0}$ so that $I_{0} \geq \bar{I}$, by using Corollary 10, provided

$$
\begin{aligned}
& I^{1 / q}(t) \\
& \quad \geq \max \left\{\frac{2 V_{1}(\varepsilon) S_{\infty}^{q-1} \gamma^{1 / p}}{\varepsilon} ; \frac{2 M V_{2}(\varepsilon) S_{\infty}^{q-1} \gamma^{1 / p}}{\varepsilon} ; 1\right\},
\end{aligned}
$$

we have

$$
\begin{aligned}
\left|\frac{\phi_{q}(y) \psi(t, x)}{I}\right| & <\frac{\sigma \varepsilon}{2}+\frac{\varepsilon}{2} ; \\
\left|\frac{\phi_{q}(y) x \psi_{x}(t, x)}{I}\right| & <\frac{\sigma \varepsilon}{2}+\frac{\varepsilon}{2},
\end{aligned}
$$

where $\sigma=C_{\infty} S_{\infty}^{q-1}$. Since $\varepsilon>0$ is arbitrary, (ii) is proved.

(iii) Set $\eta_{1}=q C_{\infty}^{p+2} S_{\infty}^{q-2}, \eta_{2}=q M^{p+2} S_{\infty}^{q-2} \gamma^{1-2 / q}, v_{1}=$ $C_{\infty}^{2} S_{\infty}^{2(q-1)}, v_{2}=M V_{2}(\varepsilon) \gamma^{2 / p} S_{\infty}^{2(q-1)}$. In view of (16), Lemma 7 (ii), and $p \geq 2$, we can get

$$
\begin{aligned}
& \left|\frac{x \psi^{2}(t, x) \phi_{q}^{\prime}(y) \phi_{p}(x)}{I^{2}}\right| \\
& \quad=\left|\frac{(q-1) x \psi^{2}(t, x) \phi_{p}(x) y^{q-2}}{I^{2}}\right| \\
& \quad \leq \frac{q|x|^{p+2}|y|^{q-2} \varepsilon^{2}}{4 \gamma^{4 / p} I^{2}}+\frac{q M^{p+2}|y|^{q-2}}{I^{2}} \\
& \quad \leq \frac{\varepsilon^{2}}{4} q I^{4 / p-2} C_{\infty}^{p+2} S_{\infty}^{q-2}+\frac{q M^{p+2} S_{\infty}^{q-2} \gamma^{1-2 / q}}{I^{1+2 / q}} \\
& \quad \leq \frac{\varepsilon^{2} \eta_{1}}{2} I^{4 / p-2}+\frac{\eta_{2}}{2 I^{1+2 / q}} ;
\end{aligned}
$$

$\left|\frac{x \psi(t, x) \psi_{x}(t, x) \phi_{q}^{2}(y)}{I^{2}}\right|$

$$
\begin{aligned}
& \leq \frac{|y|^{2(q-1)}|x|^{2} \varepsilon^{2}}{4 \gamma^{4 / p} I^{2}}+\frac{M V_{1}(\varepsilon) V_{2}(\varepsilon)|y|^{2(q-1)}}{I^{2}} \\
& \leq \frac{\varepsilon^{2}}{4} I^{4 / p-2} C_{\infty}^{2} S_{\infty}^{2(q-1)}+\frac{M V_{1}(\varepsilon) V_{2}(\varepsilon) \gamma^{2 / p} S_{\infty}^{2(q-1)}}{I^{2 / q}} \\
& \leq \frac{\varepsilon^{2} v_{1} I^{4 / p-2}}{2}+\frac{V_{1}(\varepsilon) v_{2}}{2 I^{2 / q}} ;
\end{aligned}
$$$$
\left|\frac{x^{2} \psi_{x}(t, x) \psi(t, x) \phi_{q}^{\prime}(y) \phi_{p}(x)}{I^{2}}\right|
$$$$
=\left|\frac{(q-1) x^{2} \psi(t, x) \psi_{x}(t, x) \phi_{p}(x)|y|^{q-2}}{I^{2}}\right|
$$$$
\leq \frac{q|x|^{p+2}|y|^{q-2} \varepsilon^{2}}{4 \gamma^{4 / p} I^{2}}+\frac{q M^{p+1} V_{1}(\varepsilon) V_{2}(\varepsilon)|y|^{q-2}}{I^{2}}
$$$$
\leq \frac{\varepsilon^{2}}{4} q I^{4 / p-2} C_{\infty}^{p+2} S_{\infty}^{q-2}
$$$$
+\frac{q M^{p+2} V_{1}(\varepsilon) V_{2}(\varepsilon) S_{\infty}^{q-2} \gamma^{1-2 / q}}{I^{1+2 / q}}
$$ 


$$
\begin{aligned}
& \leq \frac{\varepsilon^{2} \eta_{1}}{2} I^{4 / p-2}+\frac{V_{1}(\varepsilon) V_{2}(\varepsilon) \eta_{2}}{2 I^{1+2 / q}} \\
&\left|\frac{x^{2} \psi_{x}^{2}(t, x) \phi_{q}^{2}(y)}{I^{2}}\right| \\
& \quad \leq \frac{|x|^{2}|y|^{2(q-1)} \varepsilon^{2}}{4 \gamma^{4 / p} I^{2}}+\frac{M^{2} V_{2}(\varepsilon)|y|^{2(q-1)}}{I^{2}} \\
& \quad \leq \frac{\varepsilon^{2}}{4} I^{4 / p-2} C_{\infty}^{2} S_{\infty}^{2(q-1)}+\frac{M^{2} V_{2}(\varepsilon) \gamma^{2 / p} S_{\infty}^{2(q-1)}}{I^{2 / q}} \\
& \quad \leq \frac{\varepsilon^{2} v_{1} I^{4 / p-2}}{2}+\frac{M \nu_{2}}{2 I^{2 / q}} .
\end{aligned}
$$

Then, given $\bar{I}>0$, choosing $I_{0}$ so that $I_{0} \geq \bar{I}$, by using Corollary 10, provided

$$
\begin{gathered}
I^{1 / q}(t) \geq \max \left\{\left(\frac{\eta_{2}}{\varepsilon}\right)^{1 /(q+2)} ;\left(\frac{V_{1}(\varepsilon) \nu_{2}}{\varepsilon}\right)^{1 / 2} ;\right. \\
\left.\left(\frac{V_{1}(\varepsilon) V_{2}(\varepsilon) \eta_{2}}{\varepsilon}\right)^{1 /(q+2)} ;\left(\frac{M \nu_{2}}{\varepsilon}\right)^{1 / 2} ; 1\right\},
\end{gathered}
$$

we have

$$
\begin{aligned}
\left|\frac{x \psi^{2}(t, x) \phi_{q}^{\prime}(y) \phi_{p}(x)}{I^{2}}\right| & \leq \frac{\eta_{1} \varepsilon^{2}}{2}+\frac{\varepsilon}{2} ; \\
\left|\frac{x \psi(t, x) \psi_{x}(t, x) \phi_{q}^{2}(y)}{I^{2}}\right| & \leq \frac{v_{1} \varepsilon^{2}}{2}+\frac{\varepsilon}{2} ; \\
\left|\frac{x^{2} \psi_{x}(t, x) \psi(t, x) \phi_{q}^{\prime}(y) \phi_{p}(x)}{I^{2}}\right| & \leq \frac{\eta_{1} \varepsilon^{2}}{2}+\frac{\varepsilon}{2} ; \\
\left|\frac{x^{2} \psi_{x}^{2}(t, x) \phi_{q}^{2}(y)}{I^{2}}\right| & \leq \frac{\nu_{1} \varepsilon^{2}}{2}+\frac{\varepsilon}{2} .
\end{aligned}
$$

Since $\varepsilon>0$ is arbitrary, the proof of (iii) is finished.

$$
\begin{aligned}
& \forall t \in\left[0,2 \pi_{p}\right] \text {, set } \\
& b_{1}(t)=\frac{\partial \Phi_{1}}{\partial I}=\frac{-x\left[(p-1) \psi(t, x)-x \psi_{x}(t, x)\right]}{p^{2} I^{2}}, \\
& b_{2}(t)=\frac{\partial \Phi_{1}}{\partial \theta}=\frac{-\phi_{q}(y)}{\omega p I}\left[\psi(t, x)+x \psi_{x}(t, x)\right], \\
& b_{3}(t)=\frac{\partial \Phi_{2}}{\partial \theta} \\
& =\frac{\left[\lambda \phi_{p}\left(x^{+}\right)-\mu \phi_{p}\left(x^{-}\right)\right] \phi_{q}^{\prime}(y) \psi(t, x)}{\omega^{2}} \\
& -\frac{\phi_{q}^{2}(y) \psi_{x}(t, x)}{\omega^{2}} .
\end{aligned}
$$

As a result of Lemma 9, Corollary 10, and Lemma 12, we have the following.
Lemma 13. $\forall t, s \in\left[0,2 \pi_{p}\right]$, the following conclusions hold:

(i) $b_{1}(t)=o\left(1 / I_{0}\right)$, as $I_{0} \rightarrow+\infty$.

(ii) $b_{2}(t)=o(1)$, as $I_{0} \rightarrow+\infty$.

(iii) $b_{1}(t) \cdot b_{3}(s)=o(1)$, as $I_{0} \rightarrow+\infty$.

Let us consider the variational equation of (17) with respect to the initial value $I_{0}$. One can verify that

$$
\begin{aligned}
& \dot{\theta}_{I_{0}}=b_{1}(t) \frac{\partial I}{\partial I_{0}}+b_{2}(t) \frac{\partial \theta}{\partial I_{0}} \\
& \dot{I}_{I_{0}}=-b_{2}(t) \frac{\partial I}{\partial I_{0}}+b_{3}(t) \frac{\partial \theta}{\partial I_{0}} .
\end{aligned}
$$

Lemma 14. For all $t \in\left(0,2 \pi_{p}\right], I_{0} \rightarrow+\infty$, one has

(i) $\theta_{I_{0}}\left(t ; \theta_{0}, I_{0}\right) \rightarrow 0$;

(ii) $I_{I_{0}}\left(t ; \theta_{0}, I_{0}\right)=1+o(1)$;

(iii) $\theta_{\theta_{0}}\left(t ; \theta_{0}, I_{0}\right)=1+o(1)$.

Proof. From variational equations (41) and Lemma 13, one has

$$
\begin{aligned}
\theta_{I_{0}}(t) & =e^{\int_{0}^{t} b_{2}(s) d s} \cdot \int_{0}^{t} e^{-\int_{0}^{s} b_{2}(t) d t} b_{1}(s) \cdot I_{I_{0}}(s) d s \\
= & (1+o(1)) \int_{0}^{t} b_{1}(s) \cdot I_{I_{0}}(s) d s, \\
I_{I_{0}}(t) & =e^{-\int_{0}^{t} b_{2}(s) d s} \cdot\left(1+\int_{0}^{t} e^{\int_{0}^{s} b_{2}(t) d t} b_{3}(s) \cdot \theta_{I_{0}}(s) d s\right) \\
= & 1+o(1) \\
& +(1+o(1)) \int_{0}^{t} b_{3}(s) \cdot\left(\int_{0}^{s} b_{1}(t) \cdot I_{I_{0}}(t) d t\right) d s \\
= & 1+o(1)+o(1) \int_{0}^{t} \int_{0}^{s} I_{I_{0}}(t) d t d s,
\end{aligned}
$$

and here we have used $\theta_{I_{0}}(0)=0$ and $I_{I_{0}}(0)=1$.

Hence, for all $t \in\left(0,2 \pi_{p}\right], I_{0} \rightarrow+\infty$, we have $I_{I_{0}}(t)=$ $1+o(1)$ and $\theta_{I_{0}}(t)=(1+o(1)) \int_{0}^{t} b_{1}(s) d s \rightarrow 0$. Thus, (i) and (ii) are proved.

To prove (iii), we consider the variational equation of (17) about $\theta_{0}$; one can get

$$
\begin{aligned}
& \dot{\theta}_{\theta_{0}}=b_{1}(t) \frac{\partial I}{\partial \theta_{0}}+b_{2}(t) \frac{\partial \theta}{\partial \theta_{0}}, \\
& \dot{I}_{\theta_{0}}=-b_{2}(t) \frac{\partial I}{\partial \theta_{0}}+b_{3}(t) \frac{\partial \theta}{\partial \theta_{0}} .
\end{aligned}
$$

By using a similar argument in (ii), we can also show that $\theta_{\theta_{0}}\left(t ; \theta_{0}, I_{0}\right)=1+o(1), \forall t \in\left(0,2 \pi_{p}\right]$, as $I_{0} \rightarrow+\infty$. This completes the proof of Lemma 14.

Next, we will develop an estimate of upper bound and lower bound for $b_{1}(t)$. 
Lemma 15. Let $d \geq 0$ satisfy $\left(A_{2}\right)$.

(i) If $\left|x\left(t ; \theta_{0}, I_{0}\right)\right| \leq d$ for all $t \in\left[0,2 \pi_{p}\right]$, then there exists a constant $E_{d}>0$, such that $\left|b_{1}(t)\right| \leq E_{d} / I^{2}(t)$.

(ii) If $\left|x\left(t ; \theta_{0}, I_{0}\right)\right| \geq d$ for all $t \in\left[0,2 \pi_{p}\right]$, then there exists a constant $F_{d}>0$, such that $\left|b_{1}(t)\right| \geq F_{d} / I^{2}(t)$. Moreover, if $\left|x\left(t ; \theta_{0}, I_{0}\right)\right| \geq d$, then $b_{1}(t)<0$.

Proof. (i) If $\left|x\left(t ; \theta_{0}, I_{0}\right)\right| \leq d$, take $M_{d}=\max _{|x| \leq d, t \in\left[0,2 \pi_{p}\right]} \mid(p-$ 1) $\psi(x, t)-x \psi_{x}(x, t) \mid$. Then

$$
\begin{aligned}
\left|b_{1}(t)\right| & =\left|\frac{-x\left[(p-1) \psi(t, x)-x \psi_{x}(t, x)\right]}{p^{2} I^{2}}\right| \\
& \leq \frac{|x| M_{d}}{p^{2} I^{2}(t)} \leq \frac{d M_{d}}{p^{2} I^{2}(t)} .
\end{aligned}
$$

Writing $E_{d}=d M_{d} / p^{2}$, we have $\left|b_{1}(t)\right| \leq E_{d} / I^{2}(t)$.

(ii) If $\left|x\left(t ; \theta_{0}, I_{0}\right)\right| \geq d$, with condition $\left(A_{2}\right)$, it is easy to know that $(p-1) x \psi(t, x)-x^{2} \psi_{x}(t, x)>\beta x$ when $x \geq d$ and $(p-1) x \psi(t, x)-x^{2} \psi_{x}(t, x)>-\beta x$ when $x \leq-d$. Hence, $b_{1}(t)<0$ and

$$
\begin{aligned}
\left|b_{1}(t)\right| & =\left|\frac{-x\left[(p-1) \psi(t, x)-x \psi_{x}(t, x)\right]}{p^{2} I^{2}}\right| \\
& \geq \frac{\beta|x|}{p^{2} I^{2}(t)} \geq \frac{d \beta}{p^{2} I^{2}(t)} .
\end{aligned}
$$

Therefore, setting $F_{d}=d \beta / p^{2}$, we obtain $\left|b_{1}(t)\right| \geq F_{d} \mid$ $I^{2}(t)$. The proof is complete.

Let $b_{1}(t)=b_{1}^{+}(t)-b_{1}^{-}(t)$ with $b_{1}^{ \pm}(t)=\max \left\{ \pm b_{1}(t), 0\right\}$. To estimate that the integral of $b_{1}^{+}(t)$ on $[0,2 \pi]$ is smaller than the integral of $b_{1}^{-}(t)$ on $[0,2 \pi]$, we need the following lemma.

Lemma 16. Let $d \geq 0$ be as in Theorem 1. Define $\Delta t=\{t \in$ $\left.\left[0,2 \pi_{p}\right]|| x\left(t ; \theta_{0}, I_{0}\right) \mid \leq d\right\}$. Then there exist $\overline{I_{0}}>0, T>0$, such that

$$
|\Delta t| \leq \frac{T}{I_{0}^{1 / q}}
$$

for all $I_{0} \geq \overline{I_{0}}$.

Proof. According to Lemma 11, we see that $\Delta t \rightarrow 0$ if and only if $\Delta \theta \rightarrow 0$.

By the action-angle variables transformation (16), it is not difficult to verify that there exists $\tau>0$ such that $|\tan \Delta \theta| \leq$ $\tau d / I^{1 / q}(t)$ when $\Delta \theta \rightarrow 0$. Therefore, by using Corollary 10 , we know that there exist $\overline{I_{0}}>0, T>0$, such that

$$
|\Delta t| \leq \frac{T}{I_{0}^{1 / q}}
$$

for all $I_{0} \geq \overline{I_{0}}$. Thus, we prove Lemma 16 .

The next lemma gives the estimates of $\partial \theta\left(2 \pi_{p} ; \theta_{0}, I_{0}\right) / \partial I_{0}$ for $I_{0} \gg 1$.
Lemma 17. For $I_{0} \gg 1$, one gets $\theta_{I_{0}}\left(2 \pi_{p}\right)<0$.

Proof. The following results immediately from Corollary 10, Lemma 15, and Lemma 16:

$$
\begin{aligned}
\theta_{I_{0}} & \left(2 \pi_{p}\right)=(1+o(1)) \int_{0}^{2 \pi_{p}} b_{1}(s) d s \\
& =(1+o(1))\left(\int_{b_{1}(t) \leq 0} b_{1}(s) d s+\int_{b_{1}(t) \geq 0} b_{1}(s) d s\right) \\
& \leq-(1+o(1))\left(\frac{F_{d}}{\rho_{2}^{2} I_{0}^{2}}\left(2 \pi_{p}-|\Delta t|\right)-\frac{E_{d}}{\rho_{1}^{2} I_{0}^{2}}|\Delta t|\right) \\
& \leq(1+o(1))\left(\frac{\left(\rho_{1}^{2} F_{d}+\rho_{2}^{2} E_{d}\right) T}{\rho_{1}^{2} \rho_{2}^{2} I_{0}^{2+1 / q}}-\frac{2 \pi_{p} F_{d}}{\rho_{2}^{2} I_{0}^{2}}\right) .
\end{aligned}
$$
0 .

So, if $I_{0}^{1 / q}>\left(\rho_{1}^{2} F_{d}+\rho_{2}^{2} E_{d}\right) T / 2 \pi_{p} L_{d} \rho_{1}^{2}$, we have $\theta_{I_{0}}\left(2 \pi_{p}\right)<$

\section{Proof of Theorem 1}

Now we start to give the proof of Theorem 1.

Proof of Theorem 1. Based on Lemma 17 and the AubryMather theorem [37], we can see that the Poincaré map $P$ of system (17) is a monotone twist map when $I_{0} \gg 1$. At last, using similar arguments as in [37], we may broaden the Poincaré map $P$ to a new map $\widehat{P}$ which is a whole monotone twist homeomorphism on the cylinder $S^{1} \times \mathbb{R}$ and agree with $P$ on $\mathbf{S}^{1} \times\left[I_{0},+\infty\right)$ with a fixed constant $I_{0} \gg 1$. Hence, the existence of Aubry-Mather sets $M_{\sigma}$ of $\widehat{P}$ is ensured by the Aubry-Mather theorem due to Pei [37]. Moreover, for some small $\varepsilon_{0}>0$, all those Aubry-Mather sets with rotation number $\alpha \in\left(2 \omega \pi_{p}, 2 \omega \pi_{p}+\varepsilon_{0}\right)$ lie in the domain $\mathbf{S}^{1} \times\left[I_{0},+\infty\right)$. Therefore, they happen to be the Aubry-Mather sets of the Poincaré map of $P$. From the above discussions, we have showed the existence of Aubry-Mather sets; this implies that (1) has an Aubry-Mather type solution $u_{\alpha}(t)=$ $\left(x_{\alpha}(t), x_{\alpha}^{\prime}(t)\right)$ with rotation number $\alpha$. This completes the proof of Theorem 1 .

\section{Data Availability}

No data were used to support this study.

\section{Conflicts of Interest}

The authors declare that there are no conflicts of interest regarding the publication of this paper.

\section{Acknowledgments}

This work is supported by the National Natural Science Foundation of China (Grant no. 11461056), the Youth Natural Science Foundation of Jiangxi (Grant no. 20132BAB211008), and the Natural Science Foundation of Jiangxi Provincial Department of Education (Grant no. GJJ170926). 


\section{References}

[1] Y. Wang and L. Liu, "Positive solutions for a class of fractional 3-point boundary value problems at resonance," Advances in Difference Equations, vol. 2017, Article ID 7, 2017.

[2] X. Hao, H. Wang, L. Liu, and Y. Cui, "Positive solutions for a system of nonlinear fractional nonlocal boundary value problems with parameters and p-Laplacian operato," Boundary Value Problems, vol. 2017, Article ID 182, 2017.

[3] Y. Wang and J. Jiang, "Existence and nonexistence of positive solutions for the fractional coupled system involving generalized p-Laplacian," Advances in Difference Equations, Article ID 337, 2017.

[4] X. Hao, "Positive solution for singular fractional differential equations involving derivatives," Advances in Difference Equations, Article ID 139, 2016.

[5] Y. Guan, Z. Zhao, and X. Lin, "On the existence of positive solutions and negative solutions of singular fractional differential equations via global bifurcation techniques," Boundary Value Problems, Article ID 141, 2016.

[6] L. Liu, X. Hao, and Y. Wu, "Positive solutions for singular second order differential equations with integral boundary conditions," Mathematical and Computer Modelling, vol. 57, no. 3-4, pp. 836847, 2013.

[7] J. Jiang, L. Liu, and Y. Wu, "Positive solutions to singular fractional differential system with coupled boundary conditions," Communications in Nonlinear Science and Numerical Simulation, vol. 18, no. 11, pp. 3061-3074, 2013.

[8] J. Wu, X. Zhang, L. Liu, and Y. Wu, "Positive solutions of higherorder nonlinear fractional differential equations with changingsign measure," Advances in Difference Equations, Article ID 71, 2012.

[9] J. Jiang, L. Liu, and Y. Wu, "Positive solutions for second order impulsive differential equations with Stieltjes integral boundary conditions," Advances in Difference Equations, Article ID 124, 2012.

[10] Y. Wang, L. Liu, and Y. Wu, "Positive solutions for a class of fractional boundary value problem with changing sign nonlinearity," Nonlinear Analysis: Theory, Methods \& Applications, vol. 74, no. 17, pp. 6434-6441, 2011.

[11] X. Zhang and L. Liu, "Positive solutions for m-point boundaryvalue problems with one-dimensional p-Laplacian," Applied Mathematics and Computation, vol. 37, no. 1-2, pp. 523-531, 2011.

[12] Y. Wang, L. Liu, and Y. Wu, "Positive solutions for a nonlocal fractional differential equation," Nonlinear Analysis: Theory, Methods \& Applications, vol. 74, no. 11, pp. 3599-3605, 2011.

[13] Y. Wu and Z. Zhao, "Positive solutions for third-order boundary value problems with change of signs," Applied Mathematics and Computation, vol. 218, no. 6, pp. 2744-2749, 2011.

[14] Z. Zhao, "Nonexistence and existence of positive solutions for 2nth-order singular superlinear problems with Strum-Liouville boundary conditions," Acta Mathematica Scientia B, vol. 31, no. 4, pp. 1569-1582, 2011.

[15] Z. Zhao, "Existence of positive solutions for 2nth-order singular semipositone differential equations with Sturm-Liouville boundary conditions," Nonlinear Analysis: Theory, Methods and Applications, vol. 72, no. 3-4, pp. 1348-1357, 2010.

[16] X. Zhang, L. Liu, and Y. Wu, "Multiple positive solutions of a singular fractional differential equation with negatively perturbed term," Mathematical and Computer Modelling, vol. 55, no. 3-4, pp. 1263-1274, 2012.
[17] K. Zhang, "On a sign-changing solution for some fractional differential equations," Boundary Value Problems, Article ID 59, 2017.

[18] Y. Wang and L. Liu, "Uniqueness and existence of positive solutions for the fractional integro-differential equation," Boundary Value Problems, Article ID 12, 2017.

[19] X. Hao, N. Xu, and L. Liu, "Existence and uniqueness of positive solutions for fourth-order m-point nonlocal boundary value problems with two parameters," Rocky Mountain Journal of Mathematics, vol. 43, no. 4, pp. 1161-1180, 2013.

[20] Y. Wang, L. Liu, and Y. Wu, "Existence and uniqueness of a positive solution to singular fractional differential equations," Boundary Value Problems, Article ID 81, 2012.

[21] B. Liu, J. Li, and L. Liu, "Existence and uniqueness for an $\mathrm{m}$-point boundary value problem at resonance on infinite intervals," Computers and Mathematics with Applications, vol. 64, no. 6, pp. 1677-1690, 2012.

[22] J. Mao, Z. Zhao, and N. Xu, "The existence and uniqueness of positive solutions for integral boundary value problems," Bulletin of the Malaysian Mathematical Sciences Society, vol. 34, no. 1, pp. 153-164, 2011.

[23] Z. Q. Zhao and F. S. Li, "Existence and uniqueness of positive solutions for some singular boundary value problems with linear functional boundary conditions," Acta Mathematica Sinica, vol. 27, no. 10, pp. 2073-2084, 2011.

[24] F. Li, Z. Zhao, and Y. Chen, "Global existence uniqueness and decay estimates for nonlinear viscoelastic wave equation with boundary dissipation," Nonlinear Analysis: Real World Applications, vol. 12, no. 3, pp. 1759-1773, 2011.

[25] F. Sun, L. Liu, and Y. Wu, "Infinitely many sign-changing solutions for a class of biharmonic equation with p-Laplacian and Neumann boundary condition," Applied Mathematics Letters, vol. 73, pp. 128-135, 2017.

[26] J. Liu and Z. Zhao, "Multiple positive solutions for secondorder three-point boundary-value problems with sign changing nonlinearities," Electronic Journal of Differential Equations, vol. 152, pp. 1-7, 2012.

[27] X. Zhang, L. Liu, and Y. Wu, "Existence results for multiple positive solutions of nonlinear higher order perturbed fractional differential equations with derivatives," Applied Mathematics and Computation, vol. 219, no. 4, pp. 1420-1433, 2012.

[28] X. Hao, L. Liu, and Y. Wu, "Existence and multiplicity results for nonlinear periodic boundary value problems," Nonlinear Analysis: Theory, Methods And Applications, vol. 72, no. 9-10, pp. 3635-3642, 2010.

[29] Y. Xu and H. Zhang, "Multiple positive solutions of a boundary value problem for a class of 2 nth-order m-point singular integro-differential equations in Banach spaces," Applied Mathematics and Computation, vol. 214, no. 2, pp. 607-617, 2009.

[30] $\mathrm{Y}$. $\mathrm{Xu}$ and $\mathrm{H}$. Zhang, "Multiple positive solutions of a $\mathrm{m}$ point boundary value problem for 2nth-order singular integrodifferential equations in Banach spaces," Nonlinear Analysis: Theory, Methods and Applications, vol. 70, no. 9, pp. 3243-3253, 2009.

[31] L. Liu, X. Hao, and Y. Wu, "Unbounded solutions of secondorder multipoint boundary value problem on the half-line," Boundary Value Problems, Article ID 236560, 2010.

[32] B. Liu, L. Liu, and Y. Wu, "Unbounded solutions for three-point boundary value problems with nonlinear boundary conditions on $[0,+\infty), "$ Nonlinear Analysis: Theory, Methods and Applications, vol. 73, no. 9, pp. 2923-2932, 2010. 
[33] S. Aubry and P. Y. Le Daeron, "The discrete Frenkel-Kontorova model and its extensions. I. Exact results for the ground-states," Physica D: Nonlinear Phenomena, vol. 8, no. 3, pp. 381-422, 1983.

[34] J. N. Mather, "Existence of quasiperiodic orbits for twist homeomorphisms of the annulus," Topology, vol. 21, no. 4, pp. 457-467, 1982.

[35] M. L. Pei, "Mather sets for superlinear Duffing equations," Science in China Series A, vol. 36, no. 5, pp. 524-537, 1993.

[36] D. B. Qian, "Mather sets for sublinear duffing equations," Chinese Annals of Mathematics, Series B, vol. 15, no. 4, pp. 421434, 1994.

[37] M. L. Pei, "Aubry-Mather sets for finite-twist maps of a cylinder and semilinear Duffing equations," Journal of Differential Equations, vol. 113, no. 1, pp. 106-127, 1994.

[38] A. Capietto and B. Liu, "Quasi-periodic solutions of a forced asymmetric oscillator at resonance," Nonlinear Analysis, vol. 56, no. 1, pp. 105-117, 2004.

[39] X. Yang and K. Lo, "Quasi-periodic solutions in nonlinear asymmetric oscillations," Zeitschrift für Analysis und ihre Anwendungen, vol. 26, no. 2, pp. 207-220, 2007.

[40] G. H. Shi, "Aubry-Mather sets for relativistic oscillators with anharmonic potentials," Acta Mathematica Sinica, vol. 33, no. 3, pp. 439-448, 2017.

[41] X. Wang, "Aubry-Mather sets in semilinear asymmetric duffing equations," Advances in Difference Equations, vol. 297, pp. 1-12, 2016.

[42] S. Chow and M. Pei, "Aubry-Mather theorem and quasiperiodic orbits for time dependent reversible systems," Nonlinear Analysis: Theory, Methods and Applications, vol. 25, no. 9-10, pp. 905931, 1995.

[43] A. Capietto, W. Dambrosio, and X. Wang, "Quasi-periodic solutions of a damped reversible oscillator at resonance," Differential and Integral Equations, vol. 22, no. 9-10, pp. 1033-1046, 2009.

[44] X. Wang, "Quasiperiodic solutions for sublinear asymmetric reversible systems with a nonlinear damping and periodic forcing term," Science China Mathematics, vol. 46, no. 11, pp. 1703-1714, 2016.

[45] V. Bangert, "Mather sets for twist maps and geodesics on tori," Dynamics Reported, vol. 1, pp. 1-56, 1988.

[46] J. Moser, "Recent developments in the theory of Hamiltonian systems," SIAM Review, vol. 28, no. 4, pp. 459-485, 1986.

[47] J. N. Mather, "More Denjoy minimal sets for area preserving diffeomorphisms," Commentarii Mathematici Helvetici, vol. 60, no. 4, pp. 508-557, 1985.

[48] M. Del Pino, M. Elgueta, and R. Manásevich, "A homotopic deformation alone $\mathrm{p}$ ofa Leray-Schauder degree result and existence for $\left(\left|u^{\prime}\right|^{p-2} u^{\prime}\right)^{\prime}+f(t, u)=0, u(0)=u(T)=0, p>1$," Journal of Differential Equations, vol. 80, pp. 1-13, 1989.

[49] M. Zhang, "Nonuniform nonresonance of semilinear differential equations," Journal of Differential Equations, vol. 166, no. 1, pp. 33-50, 2000.

[50] B. Liu, "Boundedness of solutions for equations with $p$ Laplacian and an asymmetric nonlinear term," Journal of Differential Equations, vol. 207, no. 1, pp. 73-92, 2004.

[51] A. C. Lazer and D. E. Leach, "Bounded perturbations of forced harmonic oscillators at resonance," Annali di Matematica Pura ed Applicata, vol. 82, pp. 49-68, 1969. 


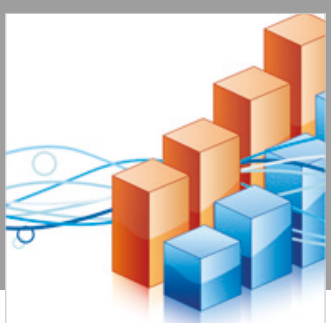

Advances in

Operations Research

\section{-n-m}
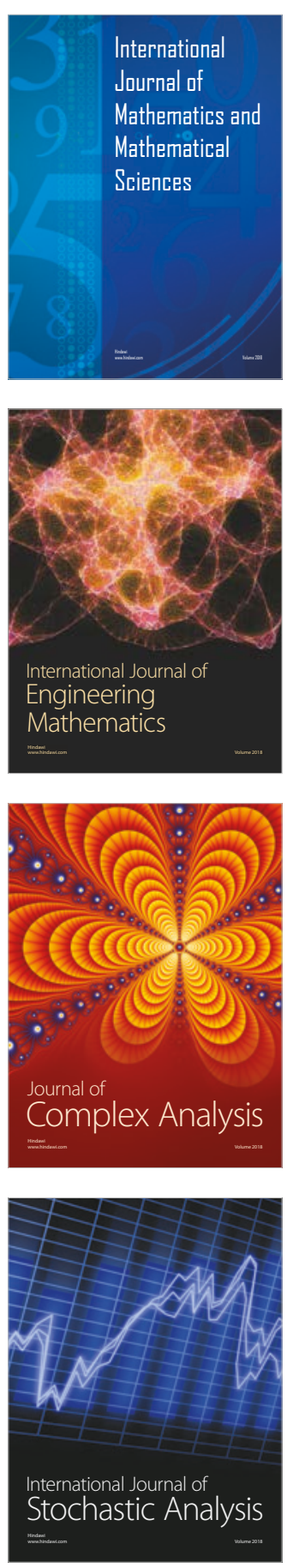
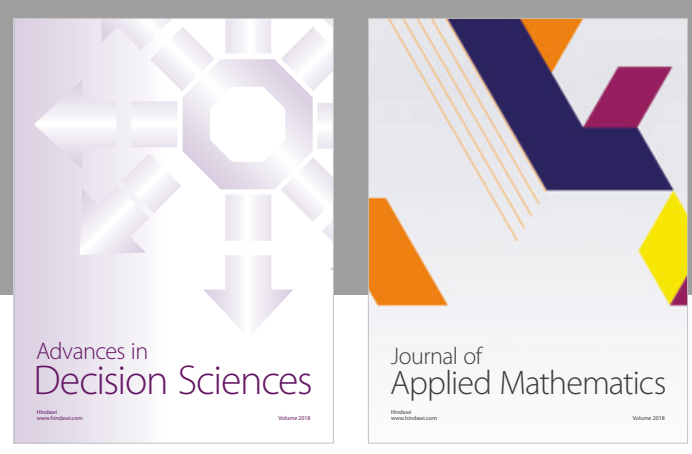

Journal of

Applied Mathematics
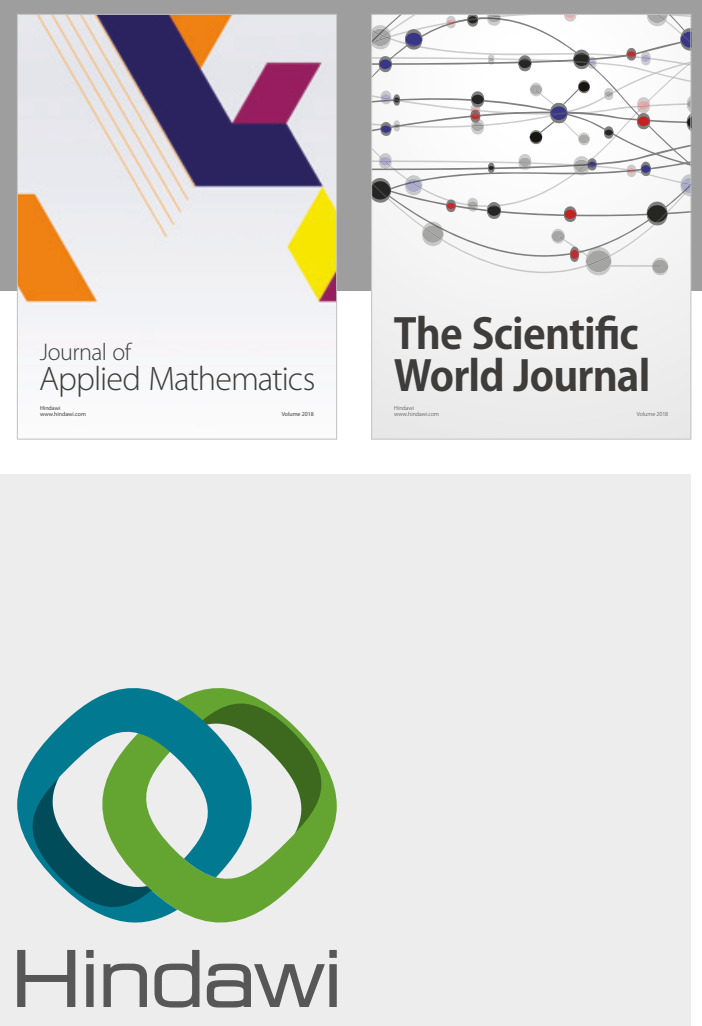

Submit your manuscripts at

www.hindawi.com

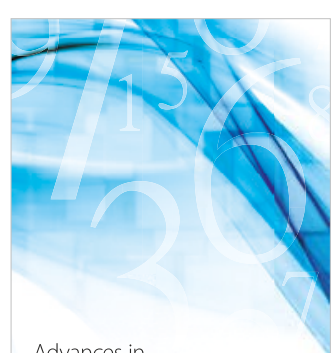

Advances in
Numerical Analysis
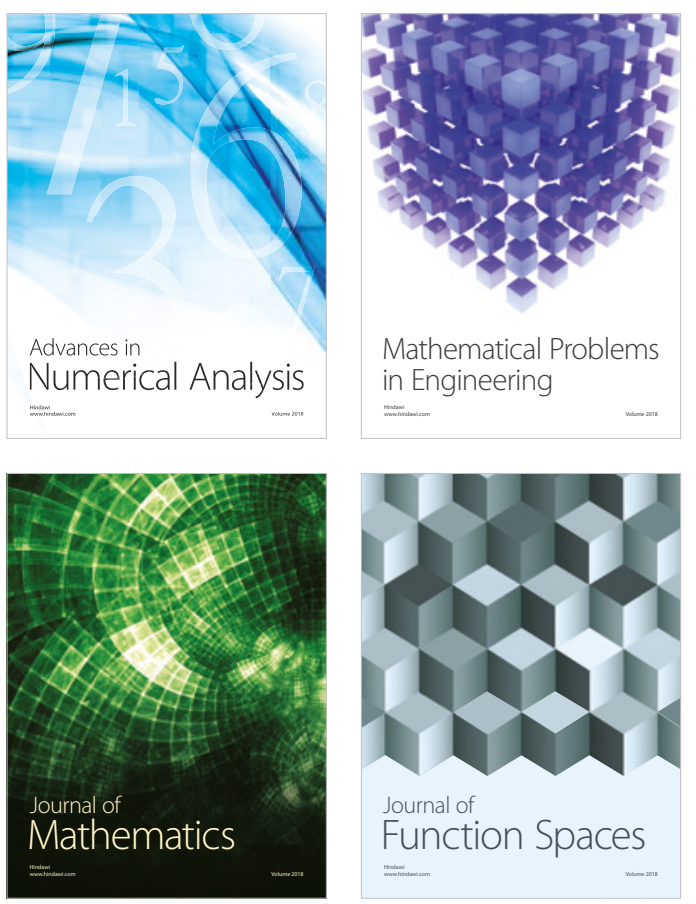

Mathematical Problems in Engineering

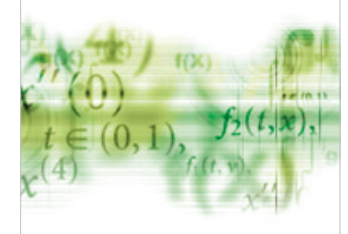

International Journal of

Differential Equations

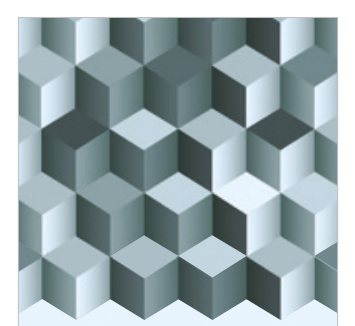

Journal of

Function Spaces

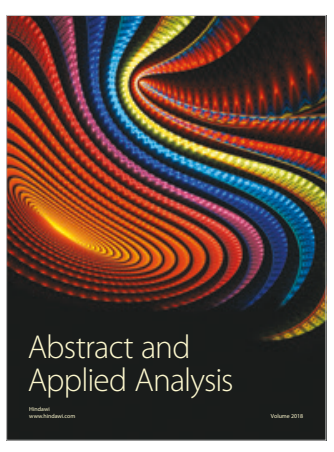

The Scientific

World Journal

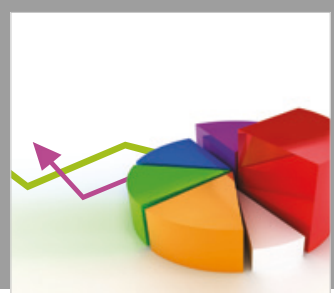

Journal of

Probability and Statistics
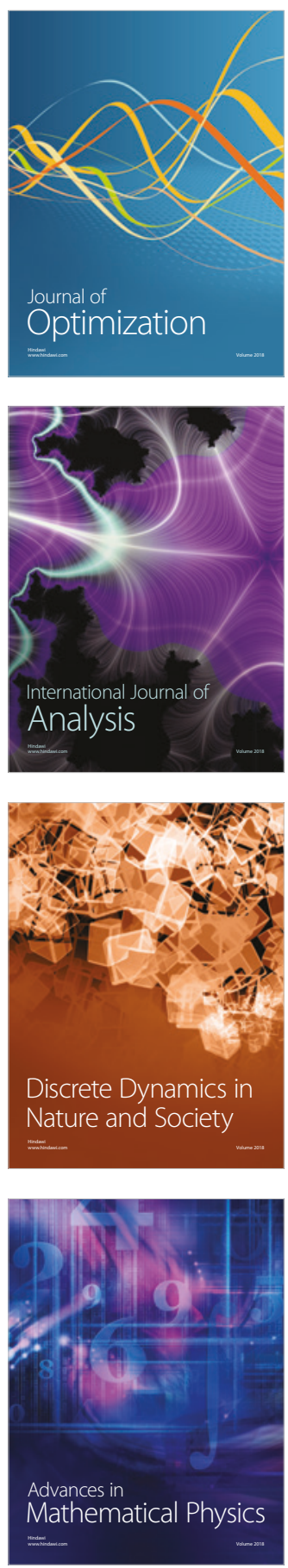